Article

\title{
Mollification Based on Wavelets
}

\section{Tohru Morita ${ }^{1}$ and Ken-ichi Sato ${ }^{2, *}$}

${ }^{1}$ Tohoku University, Sendai 980-8577, Japan; E-Mail: senmm@ jcom.home.ne.jp

${ }^{2}$ College of Engineering, Nihon University, Koriyama 963-8642, Japan

* Author to whom correspondence should be addressed; E-Mail: kensatokurume@ybb.ne.jp;

Tel.: +81-24-947-1130; Fax: +81-24-947-1130.

Received: 9 January 2013; in revised form: 11 March 2013 / Accepted: 19 March 2013 /

Published: 25 March 2013

\begin{abstract}
The mollification obtained by truncating the expansion in wavelets is studied, where the wavelets are so chosen that noise is reduced and the Gibbs phenomenon does not occur. The estimations of the error of approximation of the mollification are given for the case when the fractional derivative of a function is calculated. Noting that the estimations are applicable even when the orthogonality of the wavelets is not satisfied, we study mollifications using unorthogonalized wavelets, as well as those using orthogonal wavelets.
\end{abstract}

Keywords: mollification; Gibbs phenomenon; rapidly decaying harmonic wavelet; B-spline; Lanczos' $\sigma$-factor

\section{Introduction}

The problem of calculating the derivative of a function is an ill-posed problem, in the sense that, when a function involving noise, $f_{\epsilon}(x)$, is given instead of a smooth function $f(x)$ and the derivative $f_{\epsilon}^{\prime}(x)$ is calculated, the error is enhanced. In the numerical solution of an ill-posed problem, Murio [1] proposed the method of mollification. In that method, we calculate the mollified function $M f_{\epsilon}(x)$ by

$$
M f_{\epsilon}(x)=\left(f_{\epsilon} * \mu\right)(x):=\int_{-\infty}^{\infty} f_{\epsilon}(x-t) \mu(t) d t
$$

and we adopt $\left(M f_{\epsilon}\right)^{\prime}(x)$ as an approximate of $f^{\prime}(x)$. As the mollifier $\mu(t)$, Murio [1] uses a Gaussian probability density function. Hào et al. [2] proposed to use the de la Vallée Poussin kernel for the mollifier, in the calculation of fractional derivative. 
In a preceding paper [3], the present authors proposed to use the expansion in the orthogonal set of the rapidly decaying harmonic wavelets ( $\mathrm{rdH}$-wavelets), which were developed in [4,5]. The wavelets are characterized by a scaling function that we denote by $\phi_{1}$. When the expansion in wavelets is truncated at a stage, the truncated expansion is expressed by an expansion in a system of $\left\{\phi_{\nu}\left(x-\frac{n}{\nu}\right)\right\}_{n \in \mathbb{Z}}$, where $\phi_{\nu}(x)$ is the scaled scaling function given by $\phi_{\nu}(x)=\nu \cdot \phi_{1}(\nu x)$ for $\nu>0$, and $\mathbb{Z}$ denotes the set of all integers. Here $\nu$ is the scale at the stage of truncation. In [3], it was shown that an average of the expansion is expressed as Equation (1) if we put $\mu(x)=\mu_{\nu}(x)$ where

$$
\mu_{\nu}(x)=\int_{-\infty}^{\infty} \overline{\phi_{\nu}(y-x)} \phi_{\nu}(y) d y
$$

In [3], a series of Meyer's wavelets ([6] [p. 49]), which are special ones of the rdH-wavelets, are studied. It was shown that the use of the simplest of Meyer's wavelets agrees with the use of de la Vallée Poussin kernel adopted by Hào et al. [2].

In [2,3], the calculation of fractional derivative $D^{\lambda} f(x)$ for $\lambda>0$ was studied, which is generally an ill-posed problem. When $f_{\epsilon}$ involving noise is given in place of smooth $f$, we calculate $D^{\lambda}\left[M f_{\epsilon}\right](x)$, which approximates $D^{\lambda} f(x)$. In [2,3], we estimate how the error of this approximation can be reduced by the choice of the scale on which $\mu$ depends.

In [3], numerical examples of calculations are given, where we note that the Gibbs phenomenon is observed. In [7], seeking the wavelets for which noise is reduced and the Gibbs phenomenon is not observed, we studied this problem in the standpoint of Fourier series, where we know various attempts suppressing the Gibbs phenomenon. We took up Fejer's sum ([8] [p. 111]) and the method of Lanczos' $\sigma$-factor ([8] [p. 109ff]) and its extensions ([8] [p. 132]). Noting that Fejer's sum can be regarded as the mollification based on a special one of the $\mathrm{rdH}$-wavelets, we found one desired example. Noting that the use of the Haar wavelet is regarded as an extension of the method of Lanczos' $\sigma$-factor, we found another desired example. For those choices, the estimations of the error of approximation (EA) given in [3] do not apply. The primary purpose of the present paper is to give new estimations which apply to them.

When we look the estimations given in [3] and those given below, we find that the condition of orthogonality of the wavelets, which was used in deriving the mollification based on wavelets, is not necessary. Hence we now consider also examples of non-orthogonal set of wavelets.

In Section 2, we give a brief review of the derivation of the mollification based on orthogonal wavelets, and then give revised estimations of EA applicable to the mollifications based on the rdH-wavelets and the Haar wavelet, which are studied in [7]. In Section 3, we study these mollifications, and then mollifications based on unorthogonalized system of B-splines, in particular unorthogonalized Franklin's wavelet ([6] [p. 40]). In Section 4, we study the derivative of a function involving noise. Numerical calculations are performed by using discrete Fourier transform (DFT), which is explained in Appendix B.

We use notation $\mathbb{R}$ to represent the sets of all real numbers. We also use

$$
\begin{array}{ll}
\mathbb{R}_{>a}:=\{x \in \mathbb{R} \mid x>a\}, & \mathbb{R}_{\geq a}:=\{x \in \mathbb{R} \mid x \geq a\}, \quad \mathbb{R}_{(a, b]}:=\{x \in \mathbb{R} \mid a<x \leq b\} \\
\mathbb{Z}_{>k}:=\{n \in \mathbb{Z} \mid n>k\}, & \mathbb{Z}_{k, l}:=\{n \in \mathbb{Z} \mid k \leq n \leq l\}
\end{array}
$$


for $a, b \in \mathbb{R}$ and $k, l \in \mathbb{Z}$. For $p \in \mathbb{Z}_{>0}$, the space of those functions $f$ that $f(x)$ and $|f(x)|^{p}$ are locally integrable and integrable, respectively, on $\mathbb{R}$, is denoted by $\mathcal{L}^{p}(\mathbb{R})$. The Fourier transform of function $g(x) \in \mathcal{L}^{1}(\mathbb{R})$ is denoted by $\hat{g}(w)$ or $\hat{g}(w)$ so that

$$
\hat{g}(w)=g^{\wedge}(w)=\int_{-\infty}^{\infty} g(x) e^{-i w x} d x
$$

and $\|g\|$ and $\|g\|_{1}$ are used to denote the norms in the space $\mathcal{L}^{2}(\mathbb{R})$ and $\mathcal{L}^{1}(\mathbb{R})$, respectively. We denote the Heaviside step function by $H(x)$, so that $H(0)=\frac{1}{2}, H(-x)=0$ and $H(x)=1$ for $x \in \mathbb{R}_{>0}$.

\section{Basis of Mollification Based on Wavelets}

\subsection{Expansion in Orthogonal Wavelets}

In two recent papers [3,7], the problem of mollification is studied by using the expansion in orthogonal wavelets. We assume that $\phi_{1}$ is a scaling function of wavelets, so that (i) $\phi_{1}(x) \in \mathcal{L}^{2}\left(\mathbb{R}\right.$ ), and (ii) if $V_{k}$ for $k \in \mathbb{Z}$ is the space spanned by $\left\{2^{k / 2} \phi_{1}\left(2^{k} x-n\right)\right\}_{n \in \mathbb{Z}}$, then (a) $V_{k} \subset V_{k+1}$ for $k \in \mathbb{Z}$, (b) $\cap_{k \in \mathbb{Z}} V_{k}=0$ and (c) $\overline{\cup_{k \in \mathbb{Z}} V_{k}}=\mathcal{L}^{2}(\mathbb{R})$.

We choose a scale $\nu \in \mathbb{R}_{>0}$, and construct $\phi_{\nu}(x)$ so that its Fourier transform is given by $\hat{\phi}_{\nu}(w)=\hat{\phi}_{1}\left(\frac{w}{\nu}\right)$, and hence

$$
\phi_{\nu}(x)=\nu \cdot \phi_{1}(\nu x), \quad\left\|\phi_{\nu}\right\|^{2}=\nu\left\|\phi_{1}\right\|^{2}
$$

If $\phi_{1}(x) \in \mathcal{L}^{1}(\mathbb{R})$, we also have $\left\|\phi_{\nu}\right\|_{1}=\left\|\phi_{1}\right\|_{1}$.

We now assume that $\phi_{1}$ satisfies the following condition.

Condition $1\left\{\phi_{1}(x-n)\right\}_{n \in \mathbb{Z}}$ is an orthonormal system of functions in $\mathcal{L}^{2}(\mathbb{R})$.

Then $\left\|\phi_{1}\right\|^{2}=1$, and $\left\{\phi_{\nu}\left(x-b-\frac{n}{\nu}\right)\right\}_{n \in \mathbb{Z}}$ for fixed $b \in \mathbb{R}$ is an orthogonal system of functions. In $[3,7]$, we consider the following average with respect to $b$, of the expansion of a function $g \in \mathcal{L}^{2}(\mathbb{R})$ in this system,

$$
M_{\nu} g(x):=\nu \int_{0}^{1 / \nu}\left[\sum_{n=-\infty}^{\infty} \frac{1}{\nu} \int_{-\infty}^{\infty} \overline{\phi_{\nu}\left(t-b-\frac{n}{\nu}\right)} g(t) d t \cdot \phi_{\nu}\left(x-b-\frac{n}{\nu}\right)\right] d b
$$

We confirm that this is expressed as

$$
M_{\nu} g(x)=\left(g * \mu_{\nu}\right)(x)=\int_{-\infty}^{\infty} g(x-y) \mu_{\nu}(y) d y, \quad\left(M_{\nu} g\right)^{\wedge}(w)=\hat{g}(w) \hat{\mu}_{\nu}(w)
$$

where $\mu_{\nu}(x)$ is given by Equation (2), and hence $\hat{\mu}_{\nu}(w)=\left|\hat{\phi}_{\nu}(w)\right|^{2}=\left|\hat{\phi}_{1}\left(\frac{w}{\nu}\right)\right|^{2}$, and also $\left\|\mu_{\nu}\right\|_{1} \leq$ $\left(\left\|\phi_{\nu}\right\|_{1}\right)^{2}$ if $\phi_{1} \in \mathcal{L}^{1}(\mathbb{R})$. We define $\mu_{1}$ by $\hat{\mu}_{1}(w)=\left|\hat{\phi}_{1}(w)\right|^{2}$, and hence $\hat{\mu}_{\nu}(w)=\hat{\mu}_{1}\left(\frac{w}{\nu}\right)$. Corresponding to Equation (4), we have $\mu_{\nu}(x)=\nu \cdot \mu_{1}(\nu x)$, and $\left\|\mu_{\nu}\right\|_{1}=\left\|\mu_{1}\right\|_{1}$ if $\mu_{1} \in \mathcal{L}^{1}(\mathbb{R})$.

Lemma 1 When Condition 1 is satisfied, $\left|\hat{\phi}_{1}(0)\right|=1$.

Proof. Because of the properties (i) and (ii) of $\phi_{1}$, when Condition 1 is satisfied and $\nu$ is tended to $\infty$, $M_{\nu} g$ given by Equation (5) must converge to $g$. The second Equation of (6) shows that this requires $\lim _{\nu \rightarrow \infty} \hat{\mu}_{\nu}(w)=\left|\hat{\phi}_{1}(0)\right|^{2}=1$. 
Definition 1 Let $\mu_{1} \in \mathcal{L}^{1}(\mathbb{R})$ satisfy $\hat{\mu}_{1}(0)=1$. Let $\nu \in \mathbb{R}_{>0}$, and $\mu_{\nu}$ be given by $\hat{\mu}_{\nu}(w)=\hat{\mu}_{1}\left(\frac{w}{\nu}\right)$. Then the scale-dependent mollification $M_{\nu} g$ is defined by Equation (6), and $\mu_{1}$ and $\mu_{\nu}$ represent the mollifiers of unit scale and of scale $\nu$, respectively.

Definition 2 Let $\phi_{1}$ be the scaling function of a wavelet satisfying $\left|\hat{\phi}_{1}(0)\right|=1$. Let $\hat{\mu}_{1}(w)=\left|\hat{\phi}_{1}(w)\right|^{2}$, $\nu \in \mathbb{R}_{>0}$, and $\mu_{\nu}$ be given by $\hat{\mu}_{\nu}(w)=\hat{\mu}_{1}\left(\frac{w}{\nu}\right)$. Then we call $M_{\nu}$ g given by Equation (6) the mollification based on the wavelet, and $\mu_{1}$ and $\mu_{\nu}$ the mollifiers based on the wavelet.

Lemma $2 M_{\nu}$ g defined by Definition 1 as well as by Definition 2 approaches $g$ as $\nu \rightarrow \infty$.

Proof. By Equation (6), $\left(M_{\nu} g\right)^{\wedge}(w)=\hat{g}(w) \hat{\mu}_{1}\left(\frac{w}{\nu}\right)$. This approaches $\hat{g}(w)$ as $\nu \rightarrow \infty$.

We note here that Lemma 2 holds even when Definition 2 is adopted and the orthogonality condition given by Condition 1 is not satisfied.

\subsection{Main Results}

In the present section, we are concerned with the fractional derivative $D^{\lambda} f$ of a function $f \in \mathcal{L}^{1}(\mathbb{R})$ for $\lambda \in \mathbb{R}_{>0}$. In defining it, we use the fractional integral $D^{-\lambda} f$ for $\lambda \in \mathbb{R}_{(0,1]}$, by

$$
D^{-\lambda} f(x)=\frac{1}{\Gamma(\lambda)} \int_{-\infty}^{x}(x-t)^{\lambda-1} f(t) d t
$$

We then define the fractional derivative $D^{\lambda} f$ for $\lambda \in \mathbb{R}_{>0}$ by

$$
D^{\lambda} f(x)=D^{\lambda-m} f^{(m)}(x)
$$

when $f^{(m)}(x)=\frac{d^{m}}{d x^{m}} f(x)$ exists for $m=\lceil\lambda\rceil$. Here $\lceil\lambda\rceil$ is the least integer not less than $\lambda$, and $D^{0} f(x):=f(x)$,

Remark 1 When $f^{(m)}$ does not exist, but $f^{(m-1)}$ and $D\left[D^{\lambda-m} f^{(m-1)}(x)\right]$ exist, we put $D^{\lambda} f(x)=$ $D\left[D^{\lambda-m} f^{(m-1)}(x)\right]$, [9].

In the present subsection, we assume that $M_{\nu} g$ is defined by Definition 1.

We are interested in calculating $D^{\lambda} f$ of a function $f$ for $\lambda \in \mathbb{R}_{>0}$. When the given data is $f_{\epsilon}$, which involves noise, we mollify it as $M_{\nu} f_{\epsilon}=f_{\epsilon} * \mu_{\nu}$ and calculate $D^{\lambda}\left[M_{\nu} f_{\epsilon}\right]$ as an approximation to $D^{\lambda} f$. We estimate the error of this approximation (EA) by $\left\|D^{\lambda} f-D^{\lambda}\left[M_{\nu} f_{\epsilon}\right]\right\|$.

In [3], we considered the case where the following condition is satisfied for $l_{1}=\frac{2}{3}$.

Condition 2 There exists $l_{1} \in \mathbb{R}_{>0}$, for which $\mu_{1}$ satisfies

$$
\hat{\mu}_{1}(w)=1, \quad|w|<l_{1} \pi
$$

In [7], we considered the case where Condition 2 is not satisfied, but the following one is satisfied.

Condition 3 There exist $\alpha \in \mathbb{R}_{\geq 0}$ and $\beta, l_{1} \in \mathbb{R}_{>0}$, for which $\mu_{1}$ satisfies

$$
1-\alpha|w|^{\beta} \leq \hat{\mu}_{1}(w) \leq 1, \quad|w|<l_{1} \pi
$$


By using Condition 3 instead of Condition 2, we obtain the following propositions, in place of the propositions given in [3]. In describing them, we use the norm in the Sobolev space $\mathcal{H}^{s}(\mathbb{R})$ of order $s \in \mathbb{R}_{>0}$. When $g \in \mathcal{H}^{s}(\mathbb{R})$, the norm $\|g\|_{\mathcal{H}^{s}}$ is defined by

$$
\|g\|_{\mathcal{H}^{s}}=\left(\frac{1}{2 \pi} \int_{-\infty}^{\infty}\left(1+w^{2}\right)^{s}|\hat{g}(w)|^{2} d w\right)^{1 / 2}
$$

Proposition 1 Let Condition 3 be satisfied. Let $s, \lambda \in \mathbb{R}_{>0}$ satisfy $s>\lambda+\beta>0$, where $\beta$ is the one in Condition 3. Let $f, f_{\epsilon}$ and $D^{s} f$ belong to $\mathcal{L}^{1}(\mathbb{R})$ and $\mathcal{L}^{2}(\mathbb{R})$, and $\mu_{1}$ and $D^{\lambda} \mu_{1}$ belong to $\mathcal{L}^{1}(\mathbb{R})$. If $\left\|f-f_{\epsilon}\right\|<\epsilon\|f\|_{\mathcal{H}^{s}}$ for $\epsilon \in \mathbb{R}_{>0}$, then there exist constants $C_{1}$ and $C_{2}$, and a value of $\nu$, such that the EA is estimated as

$$
\left\|D^{\lambda} f-D^{\lambda}\left[M_{\nu} f_{\epsilon}\right]\right\|<\left(C_{1} \epsilon^{1-\lambda / s}+\alpha C_{2} \epsilon^{\beta / s}\right)\|f\|_{\mathcal{H}^{s}}
$$

Proposition 2 Let Condition 3 be satisfied. Let $s, \lambda \in \mathbb{R}_{>0}$ satisfy $s>\lambda+\beta>0$, where $\beta$ is the one in Condition 3. Let $f, f_{\epsilon}, D^{\lambda} f, f^{*}$, and $D^{s} f^{*}$ belong to $\mathcal{L}^{1}(\mathbb{R})$ and $\mathcal{L}^{2}(\mathbb{R})$, and $\mu_{1}$ and $D^{\lambda} \mu_{1}$ belong to $\mathcal{L}^{1}(\mathbb{R})$. If $\left\|f-f_{\epsilon}\right\|<\epsilon\|f\|_{\mathcal{H}^{s}},\left\|f-f^{*}\right\|<\epsilon^{\prime}\|f\|_{\mathcal{H}^{s}}$ and $\left\|D^{\lambda} f-D^{\lambda} f^{*}\right\|<\epsilon^{\prime}\|f\|_{\mathcal{H}^{s}}$ for $\epsilon, \epsilon^{\prime} \in \mathbb{R}_{>0}$, then there exist constants $C_{1}$ and $C_{2}$, and a value of $\nu$, such that the EA is estimated as

$$
\left\|D^{\lambda} f-D^{\lambda}\left[M_{\nu} f_{\epsilon}\right]\right\|<\left[\epsilon^{\prime}+C_{1}\left(\epsilon+\epsilon^{\prime}\right)^{1-\lambda / s}+\alpha C_{2}\left(\epsilon+\epsilon^{\prime}\right)^{\beta / s}\right]\|f\|_{\mathcal{H}^{s}}
$$

Proofs of these propositions are given in next subsection.

Remark 2 If we put $\alpha=0$ in Condition 3, this reduces to Condition 2, and hence Propositions 1 and 2 are valid when Condition 3 is replaced by Condition 2 and $\alpha=\beta=0$. Those are the propositions presented in [3].

\subsection{Proofs of Propositions 1 and 2}

In the following proofs, we use the following lemma, which is given, e.g., in ([10] [p. 125]).

Lemma 3 If $\mu \in \mathcal{L}^{1}(\mathbb{R})$ and $g \in \mathcal{L}^{2}(\mathbb{R})$, then $\|\mu * g\| \leq\|\mu\|_{1}\|g\|$.

Proof of Proposition 1. The EA is estimated as follows:

$$
\left\|D^{\lambda} f-D^{\lambda}\left[M_{\nu} f_{\epsilon}\right]\right\| \leq T_{1}+T_{2}+T_{3}+T_{4}
$$

where

$$
\begin{array}{cl}
T_{1}=\left\|D^{\lambda} f-D^{\lambda}\left[\Phi_{S h} f\right]\right\|, & T_{2}=\left\|D^{\lambda}\left[\Phi_{S h} f\right]-D^{\lambda}\left[M_{\nu}\left[\Phi_{S h} f\right]\right]\right\| \\
T_{3}=\left\|D^{\lambda}\left[M_{\nu}\left[\Phi_{S h} f\right]\right]-D^{\lambda}\left[M_{\nu} f\right]\right\|, & T_{4}=\left\|D^{\lambda}\left[M_{\nu} f\right]-D^{\lambda}\left[M_{\nu} f_{\epsilon}\right]\right\|
\end{array}
$$

Here $\hat{\phi}_{S h}(w)=H\left(l_{1} \pi \nu-|w|\right)$, and $\Phi_{S h} f=f * \phi_{S h}$. In [3], $T_{1}, T_{3}$ and $T_{4}$ are estimated as

$$
\begin{aligned}
& T_{1}=\left(\frac{1}{2 \pi} \int_{-\infty}^{\infty}|w|^{2 \lambda}\left(1-\hat{\phi}_{S h}(w)\right)\left(1+w^{2}\right)^{-s} \cdot\left(1+w^{2}\right)^{s}|\hat{f}(w)|^{2} d w\right)^{1 / 2} \leq \frac{1}{\left(l_{1} \pi \nu\right)^{s-\lambda}}\|f\|_{\mathcal{H}^{s}} \\
& T_{3} \leq\|\mu\|_{1} \cdot T_{1}, \quad T_{4}<\nu^{\lambda} \epsilon \cdot\left\|D^{\lambda} \mu_{1}\right\|_{1} \cdot\|f\|_{\mathcal{H}^{s}}
\end{aligned}
$$


In obtaining the above estimations for $T_{3}$ and $T_{4}$, Lemma 3 is used. In a similar way to the above evaluation of $T_{1}, T_{2}$ is estimated by using Equation (10), as

$$
\begin{aligned}
T_{2} & =\left(\frac{1}{2 \pi} \int_{-\infty}^{\infty}\left|w^{\lambda}\left[1-\hat{\mu}_{1}\left(\frac{w}{\nu}\right)\right] \hat{\phi}_{S h}(w) \hat{f}(w)\right|^{2} d w\right)^{1 / 2} \\
& \leq\left(\frac{1}{2 \pi} \int_{-l_{1} \pi \nu}^{l_{1} \pi \nu}|w|^{2 \lambda} \frac{\alpha^{2}|w|^{2 \beta}}{\nu^{2 \beta}}\left(1+w^{2}\right)^{-s} \cdot\left(1+w^{2}\right)^{s}|\hat{f}(w)|^{2} d w\right)^{1 / 2} \leq \frac{\alpha}{\nu^{\beta}}\|f\|_{\mathcal{H}^{s}}
\end{aligned}
$$

By using Equations (16) and (17) in Equation (14), we obtain

$$
\left\|D^{\lambda} f-D^{\lambda}\left[M_{\nu} f_{\epsilon}\right]\right\| \leq\left(\frac{A}{\nu^{s-\lambda}}+\frac{\alpha}{\nu^{\beta}}+B \nu^{\lambda} \epsilon\right)\|f\|_{\mathcal{H}^{s}}
$$

where $A=\left(1+\left\|\mu_{1}\right\|_{1}\right)\left(\frac{1}{l_{1} \pi}\right)^{s-\lambda}$ and $B=\left\|D^{\lambda} \mu_{1}\right\|_{1}$. The sum of the first and third terms in the right hand side is minimized when

$$
\nu=\left(\frac{(s-\lambda) A}{\lambda B \epsilon}\right)^{1 / s}
$$

Then we obtain Equation (12) with

$$
C_{1}=s\left(\frac{A}{\lambda}\right)^{\lambda / s}\left(\frac{B}{s-\lambda}\right)^{1-\lambda / s}, \quad C_{2}=\left(\frac{\lambda B}{(s-\lambda) A}\right)^{\beta / s}
$$

Proof of Proposition 2. The EA is expressed as

$$
\left\|D^{\lambda} f-D^{\lambda}\left[M_{\nu} f_{\epsilon}\right]\right\| \leq\left\|D^{\lambda} f-D^{\lambda} f^{*}\right\|+\left\|D^{\lambda} f^{*}-D^{\lambda}\left[M_{\nu} f_{\epsilon}\right]\right\|
$$

The first term on the right hand side is less than $\epsilon^{\prime} \cdot\|f\|_{\mathcal{H}^{s}}$ by assumption, and the second term is estimated as in Proposition 1 by replacing $f$ with $f^{*}$, except in $\|f\|_{\mathcal{H}^{s}}$, and $\epsilon$ by $\epsilon+\epsilon^{\prime}$, since $\left\|f^{*}-f_{\epsilon}\right\|<$ $\left(\epsilon+\epsilon^{\prime}\right) \cdot\|f\|_{\mathcal{H}^{s}}$. Hence we obtain Equation (13).

\section{Mollifiers Based on Wavelets}

We use the following three requirements in judging whether the mollifier $\mu_{\nu}$ is desirable or not. The first two were mentioned in [7], as Criterions 1 and 2.

Requirement $1 \hat{\mu}_{1}(w)$ is essentially zero for $|w|$ higher than a threshold frequency.

If this is satisfied, noise reduction is expected, since high frequency contribution is important in noise. This is concluded from Equation (6).

Requirement $2 \mu_{1}(x)$ is nonnegative for all $x \in \mathbb{R}$.

If this is satisfied, the Gibbs phenomenon does not appear.

Requirement 3 The region where $\mu_{\nu}(x)$ takes nonzero values is narrow. 
If the region is narrower, the mollified function is less smeared.

In discussing the Gibbs phenomenon, we now use the function $f_{0}(x)$ given by

$$
f_{0}(x)= \begin{cases}-1, & -1 \leq x<0 \\ 1, & 0<x \leq 1 \\ 0, & (x=0 \text { or }|x|>1)\end{cases}
$$

Requirement 2 is concluded by using the first Equation of (6) for $g=f_{0}$ when $\mu_{1}(x)$ is always nonnegative, since it follows that $\left|M_{\nu} f_{0}(x)\right| \leq 1$.

Figures 1-3 show the graphs of $\hat{\mu}_{1}(w), \mu_{1}(x)$ and $M_{8} f_{0}(x)$, for the $\mu_{1}$ that we consider in the present paper.

Figure 1. $\hat{\mu}_{1}(w)$ and $\mu_{1}(x)$ for the mollifiers based on the rdH-wavelets with $l=1$. The three curves for $u_{p}(w)$ with $p=1,2$, and 3 , are shown by thin solid, thick solid, and dashed lines, respectively.
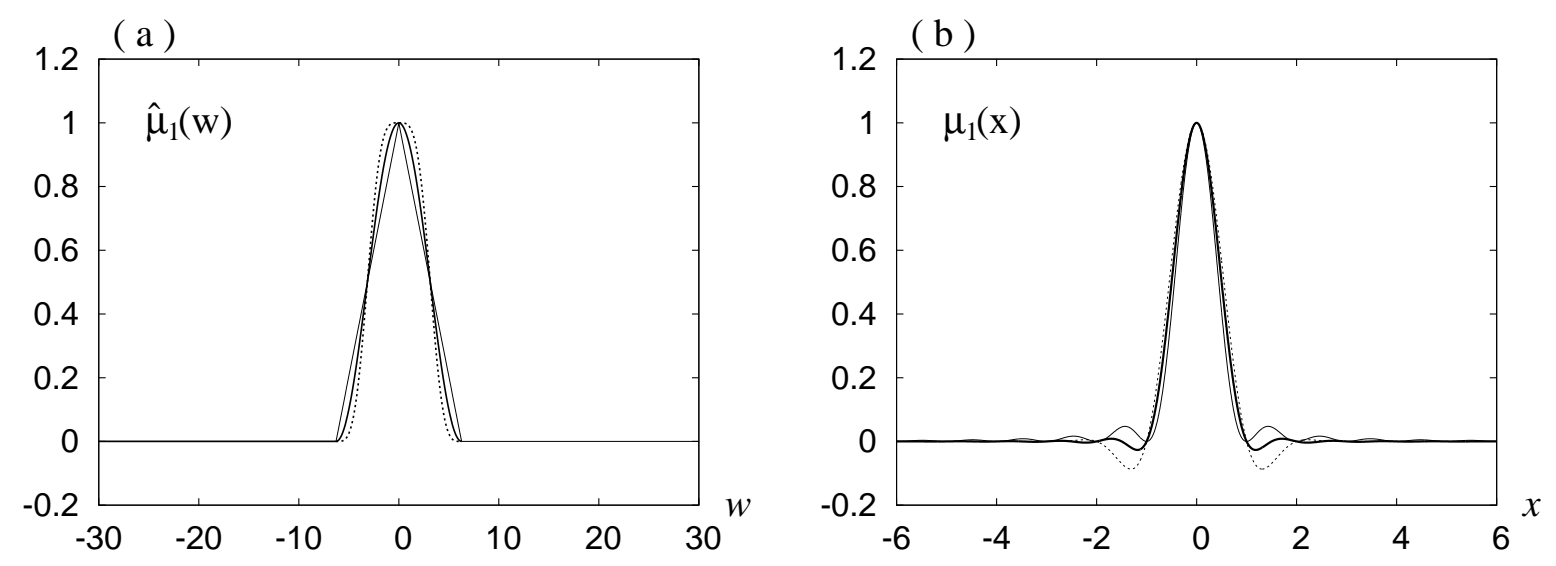

Figure 2. (a) $M_{8} f_{0}(x)$ for the mollifiers based on the rdH-wavelet with $l=1$, and (b) those based on the scaled B-spline wavelets of order $m=1,2$. In (a), the three curves for $u_{p}(w)$ with $p=1,2$, and 3 , are shown by thin solid, thick solid, and dashed lines, respectively. In (b), two curves almost overlap. $f_{0}(x)$ is also drawn both in (a) and in (b).
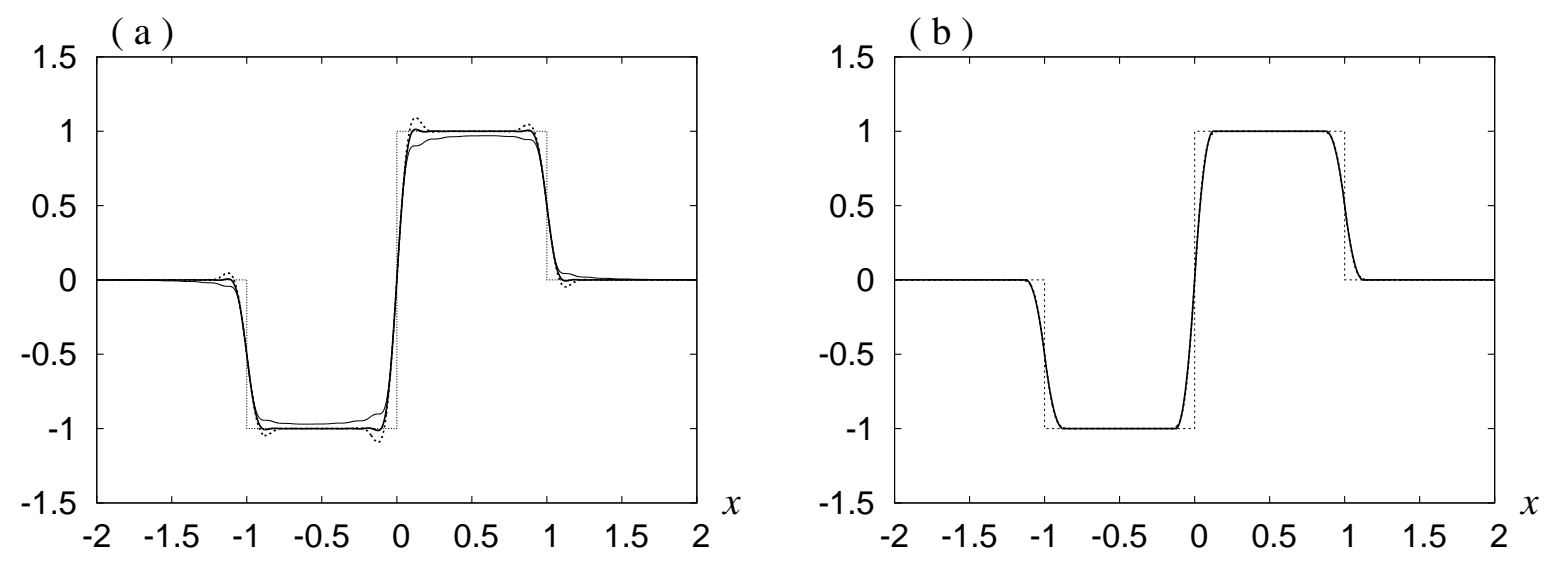
Figure 3. $\hat{\mu}_{1}(w)$ and $\mu_{1}(x)$ for the mollifiers based on the scaled B-spline wavelets of order $m=1,2,4, \infty$. The curves take greater values as $m$ increases at $w=5$ in (a), and at $x=0.2$ and $x=1.2$ in (b).
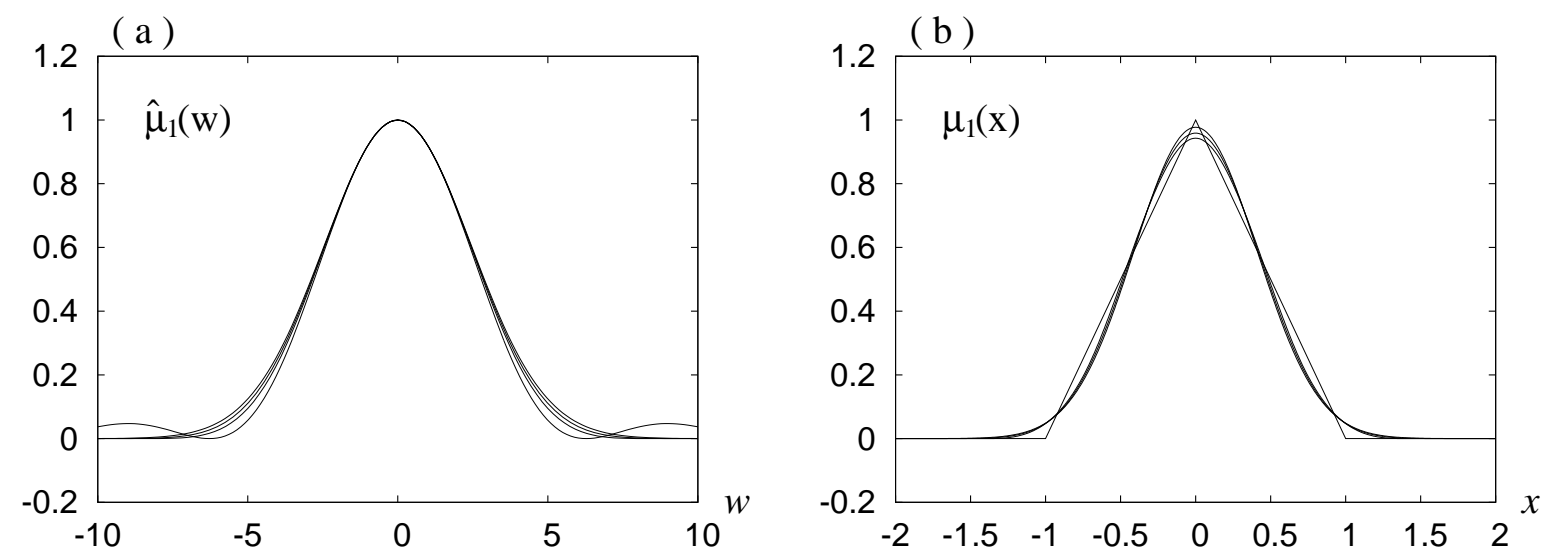

Lemma 4 If Requirement 2 is satisfied for $\mu_{1}$, which appears in Definition 1, then $\left\|\mu_{1}\right\|_{1}=\hat{\mu}_{1}(0)=1$ and hence $\mu_{1} \in \mathcal{L}^{1}(\mathbb{R})$.

\subsection{Rapidly Decaying Harmonic Wavelets}

We consider rapidly decaying harmonic (rdH-) wavelets, which were presented in [4,5]. We assume that $l \in \mathbb{R}_{(0,1]}$, and that $\hat{\phi}_{1}(w)$ is given by

$$
\hat{\phi}_{1}(w)= \begin{cases}1, & |w|<\pi-l \pi \\ u(|w|-\pi), & \pi-l \pi \leq|w| \leq \pi+l \pi \\ 0, & |w|>\pi+l \pi\end{cases}
$$

Here $u(w) \in \mathcal{L}^{2}([-l \pi, l \pi])$ is assumed to satisfy the conditions that $u(0)=\frac{1}{\sqrt{2}}, u(-l \pi)=1, u(l \pi)=0$ and $|u(w)|^{2}+|u(-w)|^{2}=1$ for $w \in \mathbb{R}_{(0, l \pi]}$.

Lemma $5 \phi_{1}$ thus defined satisfies Condition 1.

A proof of this lemma is given in Appendix A.

As $u(w)$ for $|w| \leq l \pi$, we use $u_{p}(w)$ for $p \in \mathbb{Z}_{>0}$, which are given by

$$
u_{1}(w)=\sqrt{\frac{1}{2}-\frac{1}{2 l \pi} w}, \quad u_{p+1}(w)=\sin \left(\frac{\pi}{2}\left[u_{p}(w)\right]^{2}\right), \quad p \in \mathbb{Z}_{>0}
$$

Lemma 6 For the mollifiers based on the rdH-wavelets, Requirement 1 is satisfied.

Proof. This follows from $\hat{\mu}_{1}(w)=\left|\hat{\phi}_{1}(w)\right|^{2}$ and Equation (23).

Remark 3 In [3], Meyer's wavelets were studied, where $l=\frac{1}{3}$ and $p=1,2$ and 3. In [3,7], the Gibbs phenomenon is observed in these cases.

Remark 4 If we put $l=0$ in Equation (23), $\hat{\mu}_{1}(w)=\left|\hat{\phi}_{1}(w)\right|^{2}=H(\pi-|w|)$, and then $\left(M_{\nu} g\right)^{\wedge}(w)=$ $\hat{g}(w) H(\nu \pi-|w|)$. This corresponds to truncation of Fourier series, and hence we expect the Gibbs phenomenon to occur if $\hat{g}(w)$ takes nonzero values at $|w|=\nu \pi$. 
Figures 1 and 2a show the graphs for $l=1$. In [7], it was argued that the use of $l=1$ and $u(w)=u_{1}(w)$ corresponds to the use of Fejer's sum in the Fourier series, where the Gibbs phenomenon does not appear but the convergence is not good. The curve for $u_{1}(w)$ in Figure $1 \mathrm{~b}$ shows that Requirement 2 is satisfied, and the corresponding curve in Figure 2a shows a poor convergence. The last demerit is due to the bumps outside the main peak for this case in $\mu_{1}(x)$ seen in Figure $1 \mathrm{~b}$.

Figures $1 \mathrm{~b}$ and $2 \mathrm{a}$ show that, when $l=1$ and $u(w)=u_{2}(w)$, Requirement 2 is not strictly satisfied but is almost satisfied and the Gibbs phenomenon does not appear, and the convergence is good. These figures show also that, when $l=1$ and $u(w)=u_{3}(w)$, Requirement 2 is not satisfied and the Gibbs phenomenon appears.

In [7], it was stated that the best choice is $l=1$ and $u(w)=u_{2}(w)$, where noise is reduced and the Gibbs phenomenon is not observed. In this case, $\mu_{1}$ is given by

$$
\hat{\mu}_{1}(w)=\left(\cos ^{2} \frac{w}{4}\right) H(2 \pi-|w|), \quad \mu_{1}(x)=\frac{1}{8 \pi x\left(\frac{1}{4}-x^{2}\right)} \sin (2 \pi x)
$$

and hence $\mu_{1} \in \mathcal{L}^{1}(\mathbb{R})$. Later in Section 4, we adopt this choice as Mollifier 1.

\subsection{B-Splines}

The scaling function of the Haar wavelet is given by

$$
\theta_{1}(x)=H(x) H(1-x), \quad \hat{\theta}_{1}(w)=e^{-i w / 2} \cdot \frac{\sin \frac{w}{2}}{\frac{w}{2}}
$$

We construct $\theta_{m}$ for $m \in \mathbb{Z}_{>1}$ from this $\theta_{1}$ by

$$
\theta_{m}=\theta_{m-1} * \theta_{1}, \quad \hat{\theta}_{m}(w)=\hat{\theta}_{1}(w)^{m}, \quad m \in \mathbb{Z}_{>1}
$$

This $m$ is shifted by 1 from ([6] [p. 48]). $\theta_{m+1}$ is called the B-spline of order $m$.

Use of $\phi_{1}=\theta_{m}$ for $m=1, m=2$ and $m \geq 2$ corresponds to use the Haar, the unorthogonalized (uo-) Franklin, and the uo-Battle-Lemarie wavelet ([6] [p. 40, p. 48]), respectively.

We here define a shifted Haar wavelet, by its scaling function given by

$$
\rho_{1}(x)=\theta_{1}\left(x+\frac{1}{2}\right)=H\left(\frac{1}{2}-|x|\right), \quad \hat{\rho}_{1}(w)=\frac{\sin \frac{w}{2}}{\frac{w}{2}}
$$

We now construct $\rho_{m}$ for $m \in \mathbb{Z}_{>1}$ from $\rho_{1}$ by

$$
\rho_{m}=\rho_{m-1} * \rho_{1}, \quad \hat{\rho}_{m}(w)=\hat{\rho}_{1}(w)^{m}, \quad m \in \mathbb{Z}_{>1}
$$

Adopting $\phi_{1}=\theta_{m}, \mu_{1}$ is given by

$$
\hat{\mu}_{1}(w)=\hat{\rho}_{2 m}(w)=\left(\frac{\sin \frac{w}{2}}{\frac{w}{2}}\right)^{2 m}
$$

and hence $\mu_{1}=\rho_{2 m}$. In particular, when $m=1$, we have $\mu_{1}=\rho_{2}$, where

$$
\hat{\rho}_{2}(w)=\left(\frac{\sin \frac{w}{2}}{\frac{w}{2}}\right)^{2}, \quad \rho_{2}(x)=\theta_{2}(x+1)= \begin{cases}1-|x|, & |x| \leq 1 \\ 0, & |x|>1\end{cases}
$$


When $m=2$, we have $\mu_{1}=\rho_{4}$, where

$$
\hat{\rho}_{4}(w)=\left(\frac{\sin \frac{w}{2}}{\frac{w}{2}}\right)^{4}, \quad \rho_{4}(x)=\theta_{4}(x+2)= \begin{cases}\frac{1}{6}\left[(2-|x|)^{3}-4(1-|x|)^{3}\right], & |x| \leq 1 \\ \frac{1}{6}(2-|x|)^{3}, & 1<|x| \leq 2 \\ 0, & |x|>2\end{cases}
$$

as seen in ([11] [Section 4.1]).

Lemma 7 For the mollifiers based on the B-splines, $\mu_{1}=\rho_{2 m}$, for which Requirement 2 is satisfied.

Proof. This follows from the fact that $\rho_{m}(x) \geq 0$ for all $x \in \mathbb{R}$ and $m \in \mathbb{Z}_{>0}$ by its construction.

In [7], the choice $\mu_{1}=\rho_{2}$ was studied. It was noted that the Gibbs phenomenon does not occur and noise is reduced well, though not very well. In Section 4, we adopt this as Mollifier 2.

Remark 5 In [7], the method of Lanczos' $\sigma$-factor and its multiple applications ([8] [p. 109ff, p. 132]) are called attention as a method of suppressing the Gibbs phenomenon in the Fourier series. It was noted there that Lanczos' method corresponds to the present method using $\rho_{1}$ as the mollifier. Then $\hat{\rho}_{1}(w)$ is given by Equation (28), and hence noise reduction is not expected by Requirement 1 . The extensions of Lanczos' method correspond to the present method using $\rho_{k}$ for $k \in \mathbb{Z}_{>1}$ as the mollifier. Using $\rho_{2}$ and $\rho_{4}$ corresponds to the present studies for $m=1$ and $m=2$.

\subsection{Scaled B-Splines}

In Section 3.2, the use of $\phi_{1}=\theta_{m}$ or $\rho_{m}$ for $m \in \mathbb{Z}_{>0}$ is mentioned. Then $\mu_{1}=\rho_{2 m}$, and as a probability density function (pdf), it has the variance $\sigma^{2}\left[\rho_{2 m}\right]=m \cdot \sigma^{2}\left[\rho_{2}\right]=\frac{m}{6}$, by Equation (29) and the theory of probability. In comparing two choices of $\mu_{1}$, it is desirable that the variances of them are equal or nearly equal with each other, in the respect of Requirement 3.

For $m \in \mathbb{Z}_{>0}$, we now adopt $\phi_{1}(x)=\theta_{m}(\sqrt{m} x) \sqrt{m}$ or $\phi_{1}(x)=\rho_{m}(\sqrt{m} x) \sqrt{m}$, so that $\hat{\phi}_{1}(w)=\hat{\theta}_{m}\left(\frac{w}{\sqrt{m}}\right)=\hat{\theta}\left(\frac{w}{\sqrt{m}}\right)^{m}$ or $\hat{\phi}_{1}(w)=\hat{\rho}_{m}\left(\frac{w}{\sqrt{m}}\right)=\hat{\rho}_{1}\left(\frac{w}{\sqrt{m}}\right)^{m}$. Then we have $\mu_{1}=p_{m}$, where

$$
p_{m}(x)=\rho_{2 m}(\sqrt{m} x) \sqrt{m}, \quad \hat{p}_{m}(w)=\hat{\rho}_{2 m}\left(\frac{w}{\sqrt{m}}\right)=\hat{p}_{1}\left(\frac{w}{\sqrt{m}}\right)^{m}
$$

Now $\sigma^{2}\left[p_{m}\right]=m \cdot \sigma^{2}\left[p_{1}\right] \cdot \frac{1}{m}=\frac{1}{6}$.

By the central limit theorem, as $m \rightarrow \infty, p_{m}(x)$ approaches the Gaussian pdf with the variance $\frac{1}{6}$, so that, in this limit,

$$
\mu_{1}(x)=p_{\infty}(x)=\frac{\sqrt{3}}{\sqrt{\pi}} e^{-3 x^{2}}, \quad \hat{\mu}_{1}(w)=\hat{p}_{\infty}(w)=e^{-w^{2} / 12}
$$

If we adopt $\phi_{1}(x)=\rho_{m}(\sqrt{m} x) \sqrt{m}$ for $m \in \mathbb{Z}_{>0}$, in the limit of $m \rightarrow \infty$, we have

$$
\phi_{1}(x)=\frac{\sqrt{6}}{\sqrt{\pi}} e^{-6 x^{2}}, \quad \hat{\phi}_{1}(w)=\hat{p}_{\infty}(w)^{1 / 2}=e^{-w^{2} / 24}
$$

In Figures 3a,b, the graphs of $\hat{\mu}_{1}(w)$ and $\mu_{1}(x)$, calculated by $\mu_{1}=p_{m}$ and Equations (30)-(33), are shown for $m=1,2,4$ and $\infty$. The curves for $m=3$ are not drawn. These are between the curves for 
$m=2$ and those for $m=4$, and are very close to those for $m=4$. The curves of $M_{8} f_{0}(x)$ are shown for $m=1$ and 2 in Figure $2 b$.

Figure 3 a shows that Requirement 1 is well satisfied by the curves for $m \geq 2$. Figure 3 b shows that Requirement 2 is satisfied by all the curves, and that Requirement 3 is satisfied slightly better by the curve for $m=1$. The estimations for the curves for $m \geq 2$ are nearly equal, but the best of them is for $m=2$. In Section 4, we adopt this as Mollifier 3.

\section{Numerical Computation}

We are interested in numerically calculating a function that approximates $D^{\lambda} f$. The given data are the value of $\lambda$ and a function $f_{\epsilon}$ involving noise, in place of $f$. We adopt $D^{\lambda}\left[M_{\nu} f_{\epsilon}\right]$ as an approximation of $D^{\lambda} f$, where $M_{\nu} f_{\epsilon}=f_{\epsilon} * \mu_{\nu}$. In order to calculate $D^{\lambda}\left[M_{\nu} f_{\epsilon}\right]$, we only have to choose a mollifier $\mu_{1}$ and a value of the scale $\nu$. The estimations in Section 2.2 guarantee that the error can be made small if the error $\left\|f-f_{\epsilon}\right\|$ is small. What we can practically do is to do the calculation for a number of values of $\nu$ and to choose a reasonable one among them. We show some results of such an experiment for $\lambda=1$.

In the numerical calculations, we choose sufficiently large values $L_{x}, L_{w} \in \mathbb{Z}_{>0}$ and $N=L_{x} L_{w}$, and consider discrete values of coordinates $x_{k}:=\frac{k}{L_{x}}$ for $k \in \mathbb{Z}_{-N / 2+1, N / 2}$. The integral $\int_{-\infty}^{\infty} f(x) d x$ of a function $f$ is approximated by $\sum_{k=-N / 2+1}^{N / 2} f\left(x_{k}\right) \Delta x_{k}$, where $\Delta x_{k}:=\frac{1}{L_{x}}$; See Appendix B.

We now consider the V-shaped function $f_{1}(x)$, which is given by

$$
f_{1}(x)= \begin{cases}-1+|x|, & |x| \leq 1 \\ 0, & |x|>1\end{cases}
$$

and noisy data $f_{1, \epsilon}\left(x_{k}\right)=f_{1}\left(x_{k}\right)+0.1 \cdot r_{k}$ for $k \in \mathbb{Z}$, where $r_{k}$ for each $k$ is a random number chosen from the uniform distribution in the interval $(-1,1)$. In Figure $4 \mathrm{a}, \mathrm{b}$, the functions $f_{1}(x)$ and $f_{1, \epsilon}\left(x_{k}\right)$ are shown. Figure $4 \mathrm{c}, \mathrm{d}$ shows $f_{1}^{\prime}(x)=\frac{d}{d x} f_{1}(x)$ and the central difference approximate $\dot{f}_{1, \epsilon}\left(x_{k}\right)$ given by

$$
\dot{f}_{1, \epsilon}\left(x_{k}\right):=\frac{f_{1, \epsilon}\left(x_{k+1}\right)-f_{1, \epsilon}\left(x_{k-1}\right)}{2 \Delta x_{k}}
$$

We calculate $M_{\nu} f_{1, \epsilon}(x)$ by

$$
M_{\nu} f_{1, \epsilon}(x)=\left(f_{1, \epsilon} * \mu_{\nu}\right)(x):=\sum_{k=-N / 2+1}^{N / 2} \mu_{\nu}\left(x-x_{k}\right) f_{1, \epsilon}\left(x_{k}\right) \Delta x_{k}
$$

where we put $\mu_{\nu}\left(x_{k \pm N}\right)=\mu_{\nu}\left(x_{k}\right)$ for $k \in \mathbb{R}_{(-N / 2, N / 2]}$. This quantity is differentiable and its derivative is denoted by $\left(M_{\nu} f_{1, \epsilon}\right)^{\prime}(x)$.

In Figures 5-7, $M_{\nu} f_{1}(x), M_{\nu} f_{1, \epsilon}(x),\left(M_{\nu} f_{1}\right)^{\prime}(x)$ and $\left(M_{\nu} f_{1, \epsilon}\right)^{\prime}(x)$ are shown for the following three choices of mollifier.

Mollifier 1 The mollifier based on the rdH-wavelet using $l=1$ and $u(w)=u_{2}(w)$, given by Equation (25) in Section 3.1, where $\sigma^{2}=\frac{1}{8}$ and $\sigma \fallingdotseq 0.354$.

Mollifier 2 The mollifier based on the Haar wavelet, given by $\mu_{1}=\rho_{2}$ in Section 3.2, where $\rho_{2}$ is given by Equation (31), and $\sigma^{2}=\frac{1}{6}$ and $\sigma \fallingdotseq 0.408$. 
Mollifier 3 The mollifier based on the scaled uo-Franklin wavelet, given by $\mu_{1}=p_{2}$ in Section 3.3, where $p_{2}$ is given by (33) and (32), and $\sigma^{2}=\frac{1}{6}$ and $\sigma \fallingdotseq 0.408$.

Figure 4. (a) $f_{1}(x)$; (b) $f_{1, \epsilon}\left(x_{k}\right)$; (c) $f_{1}^{\prime}(x)$; (d) $\dot{f}_{1, \epsilon}\left(x_{k}\right)$.
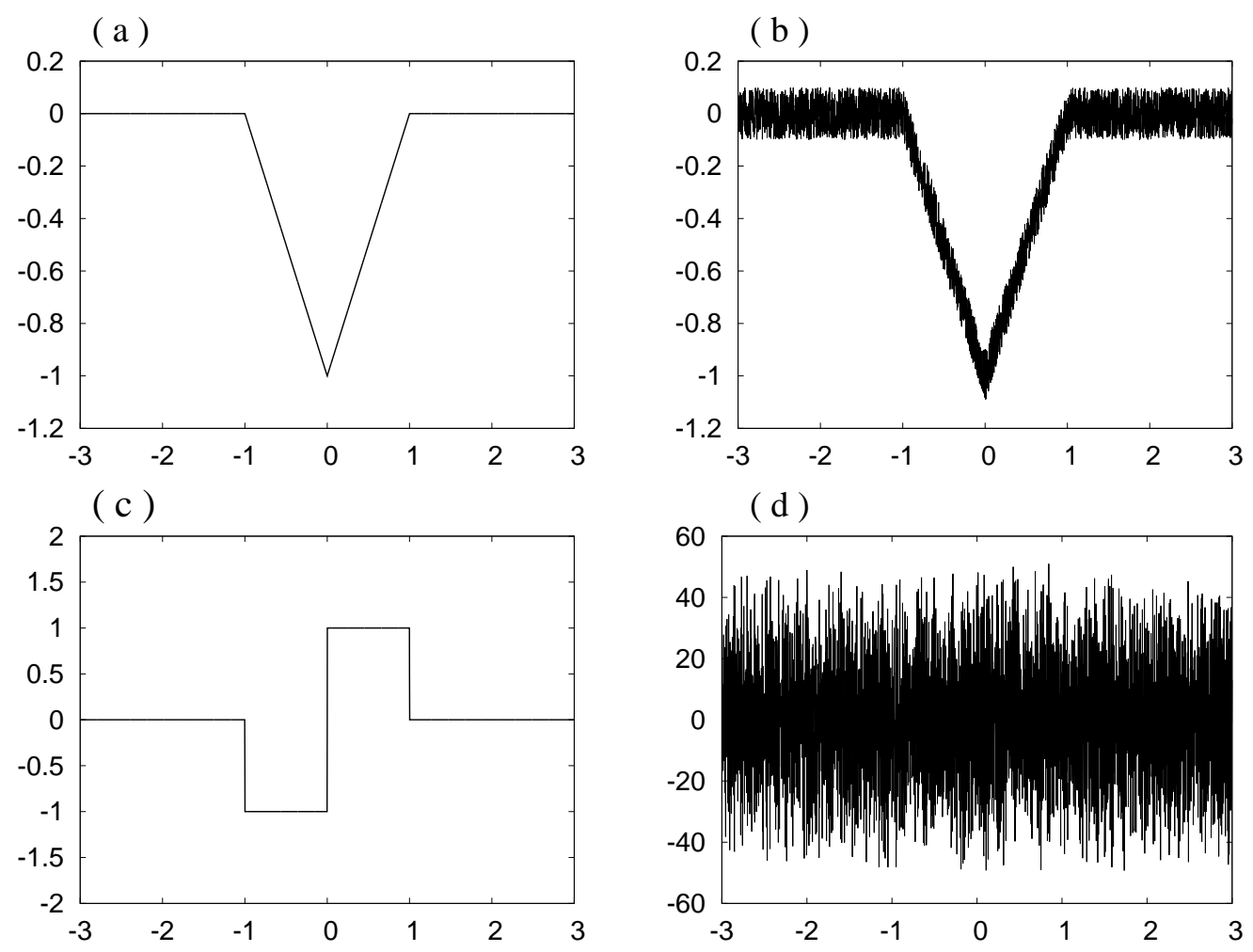

Here $\sigma^{2}$ denotes the variance of the mollifier. In each of Figures 5-7, (a) and (b) are for $\nu=16$, (c) and (d) are for $\nu=8$, and (e) and (f) are for $\nu=4$. We do not observe the Gibbs phenomenon in these curves. The noise is reduced as $\nu$ decreases.

As $\nu$ decreases, the noise is depressed, but also the bottom in (a), (c) and (e) becomes rounder, and the slope at $x_{k}=0$ in (b), (d) and (f) becomes smaller. Hence in order to see the original forms shown in Figure 4a,c clearly, we have to draw the curves for $\nu=16, \nu=8$ and $\nu=4$. By Requirement 3, this smearing is governed by the width of the mollifier $\mu_{\nu}(x)$. The width may be estimated by the value of the standard deviation $\sigma$. The value is slightly smaller for the first choice.

When we compare Figures 5-7, we do not observe difference between Figures 5 and 7. In Figure 6, we observe that the noise reduction is not so good as in the other two.

In Figure 8, we show the curves for the choice specified by $l=\frac{1}{3}$ and $u(w)=u_{2}(w)$ for the rdH-wavelet. In this case, the Gibbs phenomenon is clearly seen, which is expected in Remark 3. 
Figure 5. (a), (c), (e): $M_{\nu} f_{1}(x), M_{\nu} f_{1, \epsilon}(x) ;(\mathbf{b}),(\mathbf{d}),(\mathbf{f}):\left(M_{\nu} f_{1}\right)^{\prime}(x)$ and $\left(M_{\nu} f_{1, \epsilon}\right)^{\prime}(x)$, for Mollifier 1. The thinner curves show $M_{\nu} f_{1}(x)$ and $\left(M_{\nu} f_{1}\right)^{\prime}(x)$.
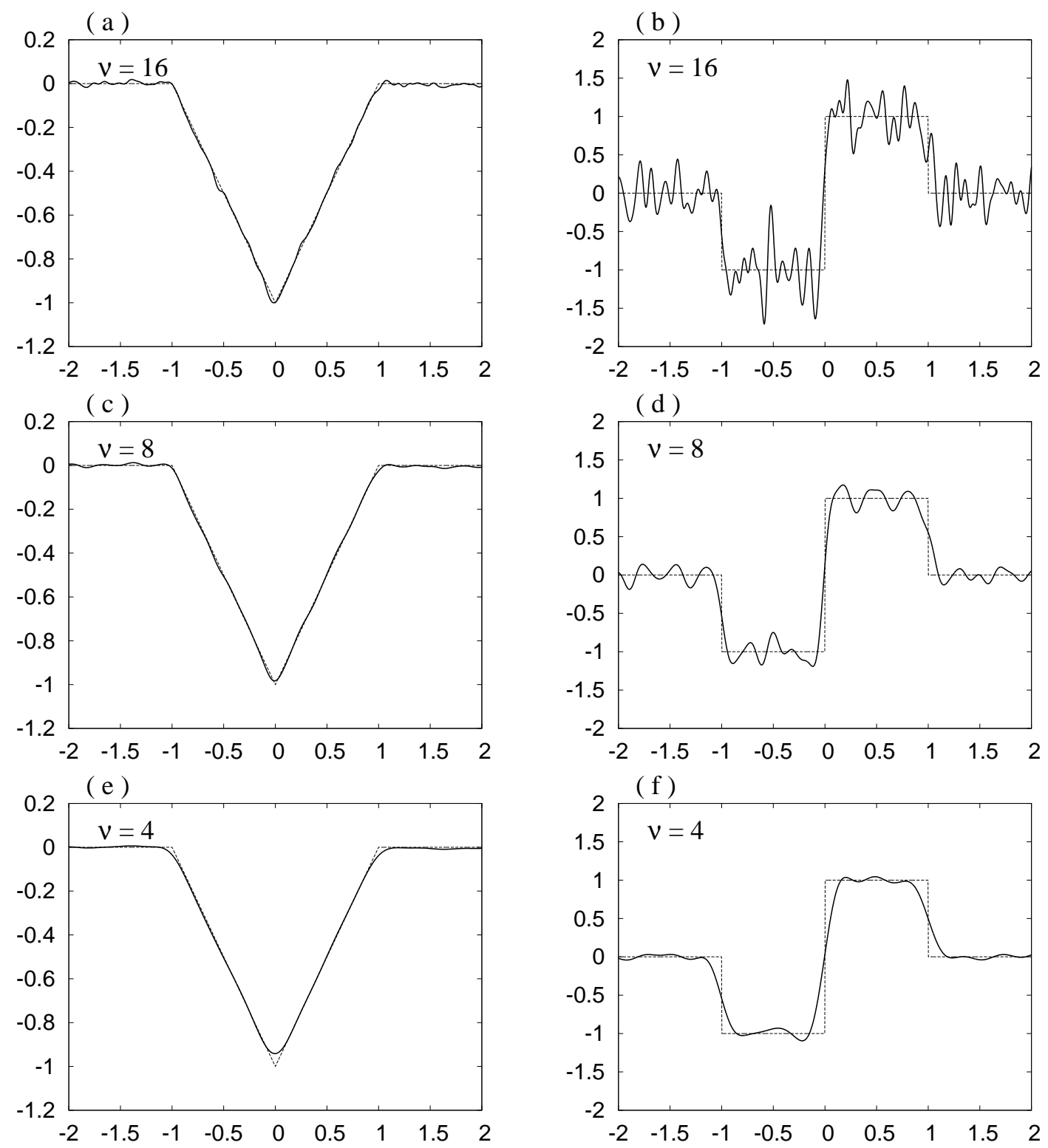

Figure 6. (a), (c), (e): $M_{\nu} f_{1}(x), M_{\nu} f_{1, \epsilon}(x) ;(\mathbf{b}),(\mathbf{d}),(\mathbf{f}):\left(M_{\nu} f_{1}\right)^{\prime}(x)$ and $\left(M_{\nu} f_{1, \epsilon}\right)^{\prime}(x)$, for Mollifier 2. The thinner curves show $M_{\nu} f_{1}(x)$ and $\left(M_{\nu} f_{1}\right)^{\prime}(x)$.
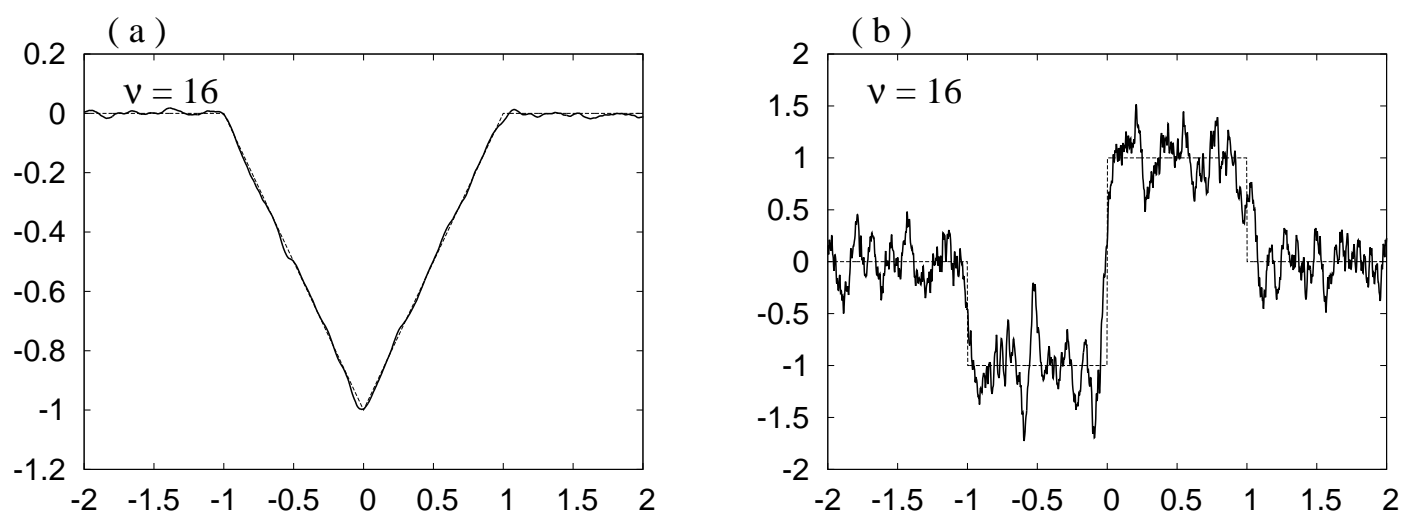
Figure 6. Cont.
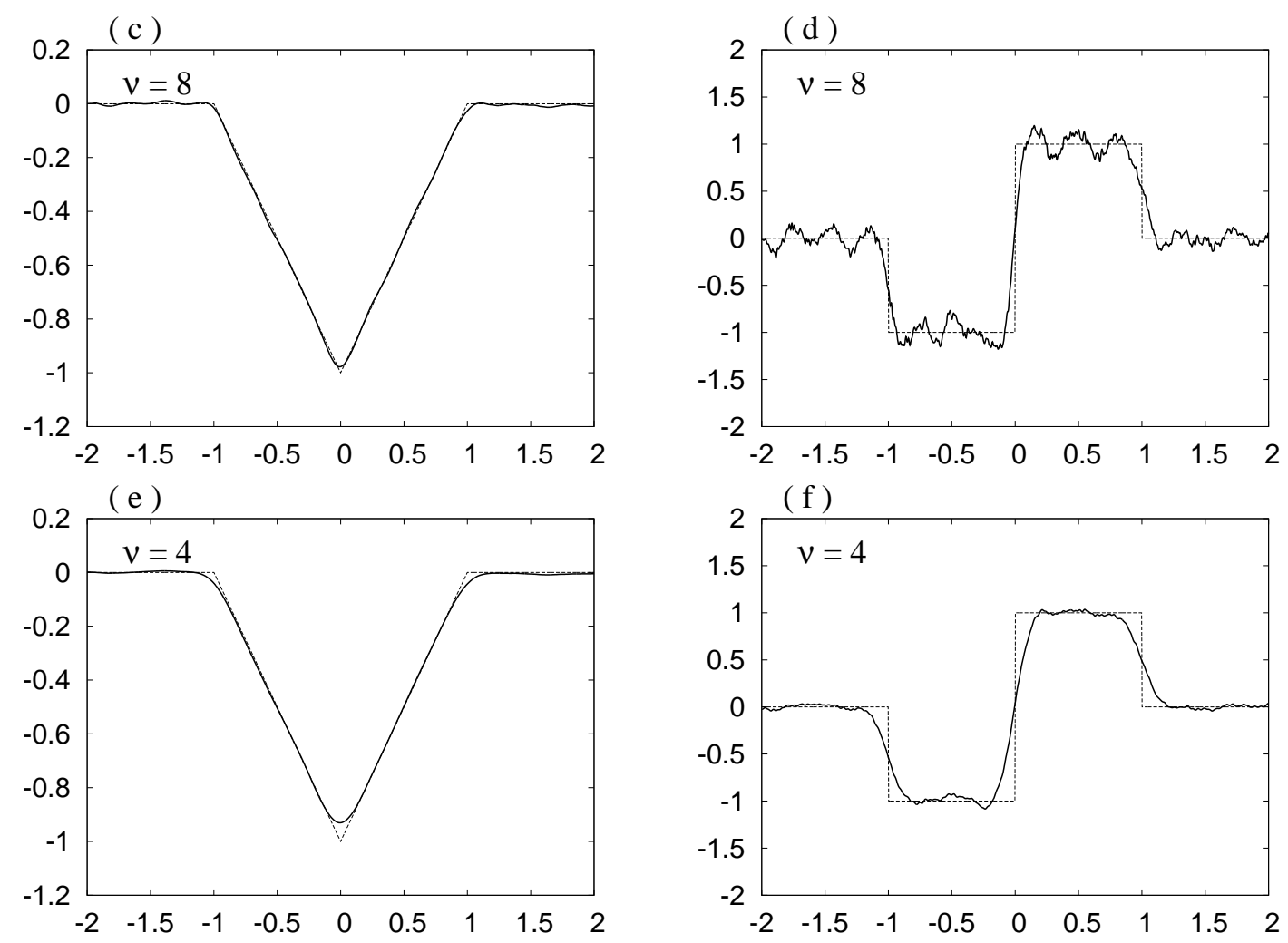

Figure 7. (a), (c), (e): $M_{\nu} f_{1}(x), M_{\nu} f_{1, \epsilon}(x) ;(\mathbf{b}),(\mathbf{d}),(\mathbf{f}):\left(M_{\nu} f_{1}\right)^{\prime}(x)$ and $\left(M_{\nu} f_{1, \epsilon}\right)^{\prime}(x)$, for Mollifier 3. The thinner curves show $M_{\nu} f_{1}(x)$ and $\left(M_{\nu} f_{1}\right)^{\prime}(x)$.
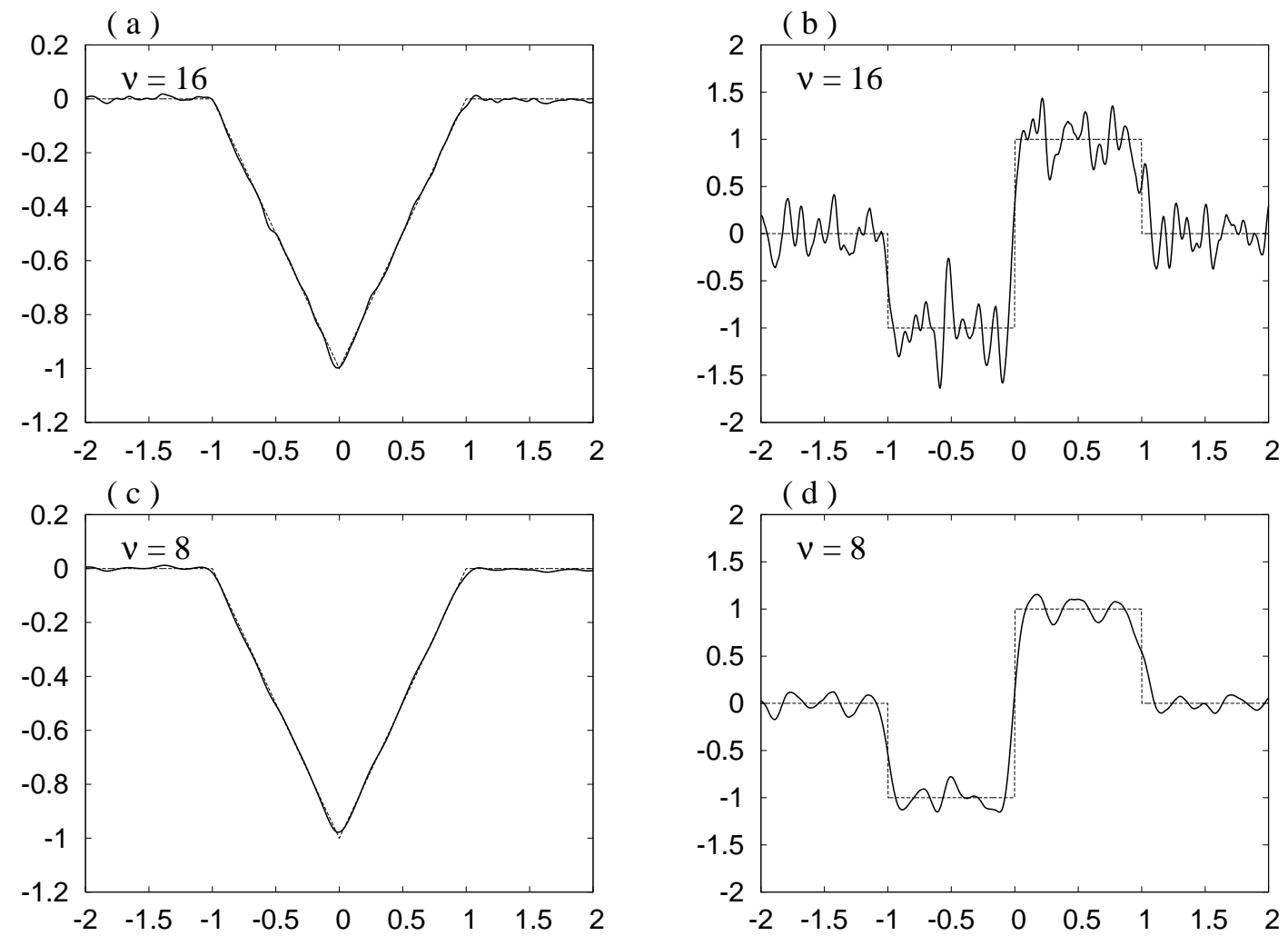
Figure 7. Cont.
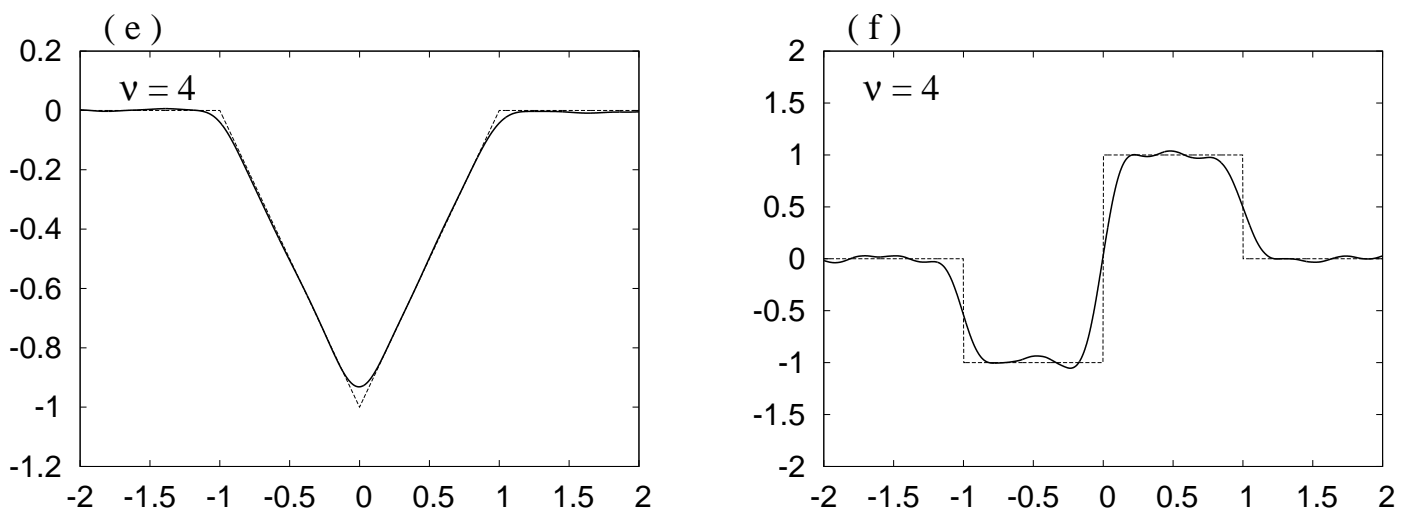

Figure 8. (a), (c), (e): $M_{\nu} f_{1}(x), M_{\nu} f_{1, \epsilon}(x) ;(\mathbf{b}),(\mathbf{d}),(\mathbf{f}):\left(M_{\nu} f_{1}\right)^{\prime}(x)$ and $\left(M_{\nu} f_{1, \epsilon}\right)^{\prime}(x)$, for the mollifier $\mu_{\nu}(x)$ based on the rdH-wavelet using $l=\frac{1}{3}$ and $u(w)=u_{2}(w)$. The thinner curves show $M_{\nu} f_{1}(x)$ and $\left(M_{\nu} f_{1}\right)^{\prime}(x)$.
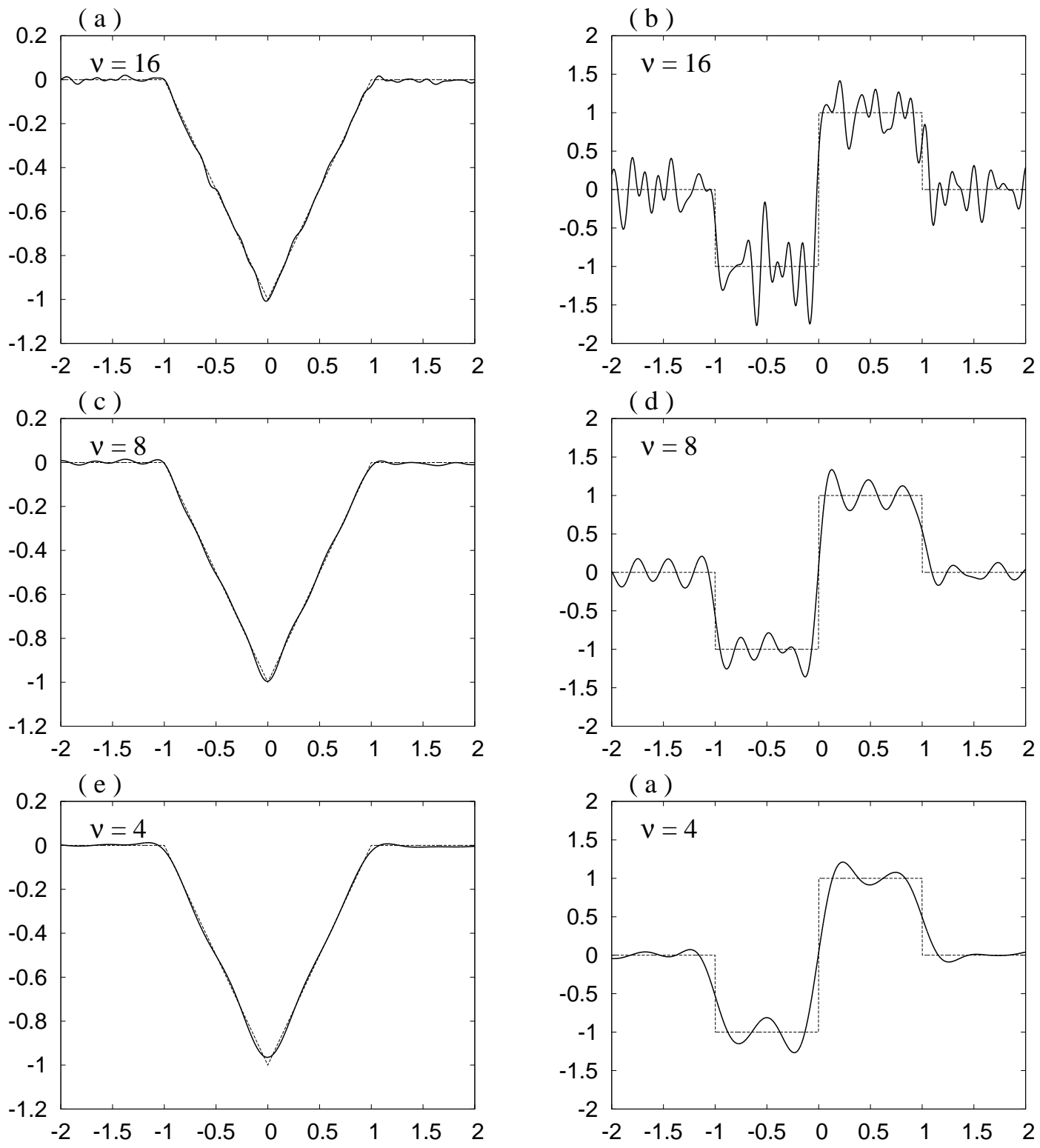


\section{Concluding Remarks}

In Section 4, we study three mollifiers called Mollifiers 1, 2 and 3. In [3], propositions are given for estimating the fractional derivative of a function, when the function given involves noise. Noting that those propositions are not applicable to the three mollifiers, we present revised propositions in Section 2. We here note that the new estimations are applicable to the three mollifiers.

We first confirm that Condition 3 is applicable to Mollifiers 1, 2 and 3, by choosing $\beta$ satisfying $0<\beta \leq 2,0<\beta \leq 1$ and $0<\beta \leq 2$, respectively. We next confirm that the conditions $\mu_{1}$, $D^{\lambda} \mu_{1} \in \mathcal{L}^{1}(\mathbb{R})$ in Propositions 1 and 2 are satisfied if $\lambda$ satisfies $\lambda>0,0<\lambda<2$ and $0<\lambda<4$, respectively, for Mollifiers 1, 2 and 3.

The last fact for Mollifier 2 is confirmed by noting that $D^{\lambda} \mu_{1}$ exists only when $\lambda<2$ for $\mu_{1}(x)=\rho_{2}(x)$ given by Equation (31), since we then have

$$
D^{\lambda} \mu_{1}(x)=\frac{1}{\Gamma(2-\lambda)}\left[(x+1)^{1-\lambda} H(x+1)-2 x^{1-\lambda} H(x)+(x-1)^{1-\lambda} H(x-1)\right]
$$

The corresponding calculation for Mollifier 3 is done by using $\mu_{1}(x)=p_{2}(x)=\rho_{4}(\sqrt{2} x) \sqrt{2}$ with the aid of Equation (32).

In the present method of mollification, we remove high frequency component of data, regarding it to be noise. Hence if noise involves low frequency component, it will not be removed, and if high frequency component in the data is not desired to be erased, the present method will not be useful.

In the present paper, we study an example of calculating the first order derivative. In [3], the derivative of order $1 / 2$ is calculated as an example, where the mollification based on an orthogonal rapidly decaying wavelet is used.

\section{Acknowledgements}

The authors are grateful to the reviewers for helpful comments and valuable suggestions. With the help of them, the authors could improve the descriptions in this paper.

\section{Appendices}

\section{A. Proof of Lemma 5}

We denote $\phi_{1, m}(x):=\phi_{1}(x-m)$ for $m \in \mathbb{Z}$. Condition 1 states that $\left(\phi_{1, m}, \phi_{1, m^{\prime}}\right)=\delta_{m, m^{\prime}}$ for $m, m^{\prime} \in \mathbb{Z}$. By using Equation (23) and $\Delta:=m-m^{\prime}$, we prove this as follows.

$$
\begin{aligned}
\left(\phi_{1, m}, \phi_{1, m^{\prime}}\right) & =\frac{1}{2 \pi} \int_{-\infty}^{\infty}\left|\hat{\phi}_{1}(w)\right|^{2} e^{i w \Delta} d w \\
& =\frac{1}{2 \pi}\left\{\int_{-l \pi}^{l \pi} u(-s)^{2} e^{i(-\pi+s) \Delta} d s+\int_{-\pi+l \pi}^{\pi-l \pi} e^{i w \Delta} d w+\int_{-l \pi}^{l \pi} u(s)^{2} e^{i(\pi+s) \Delta} d s\right\} \\
& =\frac{1}{2 \pi}\left\{\int_{-\pi+l \pi}^{\pi-l \pi} e^{i w \Delta} d w+\int_{-l \pi}^{l \pi} e^{i(\pi+s) \Delta} d s\right\}=\frac{1}{2 \pi} \int_{-\pi+l \pi}^{\pi+l \pi} e^{i w \Delta} d w=\delta_{m, m^{\prime}}
\end{aligned}
$$




\section{B. Use of Discrete Fourier Transform (DFT)}

In ([12] [Section 12.1]), description is given on the discrete Fourier transform (DFT) and its inverse. It is assumed that $N$ data $h_{k}$ for $k \in \mathbb{Z}_{0, N-1}$ are given. Then the DFT $H_{n}$ of $h_{k}$ for $n \in \mathbb{Z}_{0, N-1}$ is introduced by the first of the following equations:

$$
H_{n}=\sum_{k=0}^{N-1} h_{k} e^{2 \pi i k n / N}, \quad h_{k}=\frac{1}{N} \sum_{n=0}^{N-1} H_{n} e^{-2 \pi i k n / N}
$$

The second equation represents $h_{k}$ by the inverse DFT. In ([12] [Section 12.2]), the fast Fourier transform (FFT) algorithm is presented for the numerical computation of $\left\{H_{n}\right\}$ from $\left\{h_{k}\right\}$, and vice versa.

In the present paper, we choose two integers $L_{x} \in \mathbb{Z}_{>0}$ and $L_{w} \in \mathbb{Z}_{>0}$, and put $N:=L_{x} L_{w}$. For a function $g(x) \in \mathcal{L}^{1}(\mathbb{R})$, we consider $N$ values $g\left(x_{k}\right)$ at $x_{k}:=k / L_{x}$ for $k \in \mathbb{Z}_{-N / 2+1, N / 2}$. Then $g\left(x_{k}\right)$ is assumed to be a periodic series of $k$ with period $N$. We now put

$$
h_{k}=g\left(x_{k}\right), \quad H_{n}=\hat{g}\left(-w_{n}\right) / \Delta x, \quad w_{n}=-2 \pi n / L_{w}
$$

where $\Delta x=\frac{1}{L_{x}}$. Then $h_{k}$ and $H_{n}$ are periodic series of $k$ and $n$ with period $N$, and the formulas in (B.1) are reduced to

$$
g\left(x_{k}\right)=\frac{1}{2 \pi} \sum_{n=-N / 2+1}^{N / 2} \hat{g}\left(w_{n}\right) e^{i w_{n} x_{k}} \Delta w_{n}, \quad \hat{g}\left(w_{n}\right)=\sum_{k=-N / 2+1}^{N / 2} g\left(x_{k}\right) e^{-i w_{n} x_{k}} \Delta x_{k}
$$

where $\Delta w_{n}=2 \pi / L_{w}$ and $\Delta x_{k}=1 / L_{x}$. By this definition, $\hat{g}\left(w_{n}\right)$ is a periodic series of $n$ with period $N$.

\section{References}

1. Murio, D.A. The Mollification Method and the Numerical Solution of Ill-posed Problems; J. Wiley: New York, NY, USA, 1993.

2. Hào, D.N.; Reinhardt, H.J.; Seiffarth, F. Stable numerical differentiation by mollification. Numer. Funct. Anal. Optim. 1994, 15, 635-659.

3. Morita, T.; Sato, K. Mollification of fractional derivatives using rapidly decaying harmonic wavelet. Fract. Calc. Appl. Anal. 2011, 14, 284-300.

4. Morita, T. Rapidly decaying harmonic wavelet expansion. Interdiscip. Inf. Sci. 2008, 14, 89-101.

5. Morita, T.; Kaneko, M. Harmonic wavelet analysis of sound. Interdiscip. Inf. Sci. 2008, 14, 245-253.

6. Walter, G.G.; Shen, X. Wavelets and Other Orthogonal Systems, 2nd ed.; Chapman \& Hall/CRC: Boca Raton, FL, USA, 2001.

7. Morita, T.; Sato, K. Mollification of the Gibbs Phenomenon Using Orthogonal Wavelets. In Proceedings of the Multimedia Technology (ICMT), 2011 International Conference, Hangzhou, China, 26-28 July 2011; Volume 7, pp. 6441-6444.

8. Hamming, R.W. Digital Filters; Dover Publications Inc.: Mineola, NY, USA, 1998.

9. Morita, T.; Sato, K. Analyticity and Asymptotics of Fractional Differintegrations. In preparation.

10. Friedlander, G.; Joshi, M. Introduction to the Theory of Distributions, 2nd ed.; Cambridge U.P.: Cambridge, UK, 1998. 
11. Chui, C.K. An Introduction to Wavelets; Academic Press, Inc.: New York, NY, USA, 1992.

12. Press, W.H.; Teukolsky, S.A.; Vertterling, W.T.; Flannery, B.P. Numerical Recipes, 3rd ed.; Cambridge U.P.: Cambridge, UK, 2007.

(c) 2013 by the authors; licensee MDPI, Basel, Switzerland. This article is an open access article distributed under the terms and conditions of the Creative Commons Attribution license (http://creativecommons.org/licenses/by/3.0/). 